\title{
Deficiency of cathepsin $S$ reduces atherosclerosis in LDL receptor-deficient mice
}

\author{
Galina K. Sukhova, ${ }^{1}$ Yaou Zhang, ${ }^{2}$ Jie-Hong Pan, ${ }^{2}$ Youichiro Wada, ${ }^{3}$ Takashi Yamamoto, ${ }^{3}$ \\ Makoto Naito, ${ }^{3}$ Tatsuhiko Kodama, ${ }^{3}$ Sotirios Tsimikas, ${ }^{4}$ Joseph L. Witztum, ${ }^{4}$ \\ Michael L. Lu, ${ }^{1}$ Yasuhiko Sakara, ${ }^{1}$ Michael T. Chin, ${ }^{1}$ Peter Libby, ${ }^{1}$ and Guo-Ping Shi ${ }^{2}$ \\ ${ }^{1}$ The Leducq Center for Cardiovascular Research, Department of Medicine, Brigham and Women's Hospital and Harvard \\ Medical School, Boston, Massachusetts, USA \\ ${ }^{2}$ Department of Medicine, University of California, San Francisco, San Francisco, California, USA \\ ${ }^{3}$ Department of Molecular Biology and Medicine, University of Tokyo, Tokyo, Japan \\ ${ }^{4}$ Department of Medicine, University of California at San Diego, La Jolla, California, USA
}

Human atherosclerotic lesions overexpress the lysosomal cysteine protease cathepsin S (Cat S), one of the most potent mammalian elastases known. In contrast, atheromata have low levels of the endogenous Cat $S$ inhibitor cystatin $C$ compared with normal arteries, suggesting involvement of this protease in atherogenesis. The present study tested this hypothesis directly by crossing Cat $\mathrm{S}$-deficient $\left(\mathrm{CatS}^{-/-}\right)$mice with $\mathrm{LDL}$ receptor-deficient $\left(L D L R^{-/}\right)$mice that develop atherosclerosis on a high-cholesterol diet. Compared with $L D L R^{-/-}$mice, double-knockout mice $\left(\mathrm{CatS}^{-/-} L D L R^{-/-}\right)$developed significantly less atherosclerosis, as indicated by plaque size (plaque area and intimal thickening) and stage of development. These mice also had markedly reduced content of intimal macrophages, lipids, smooth muscle cells, collagen, $\mathrm{CD} 4^{+} \mathrm{T}$ lymphocytes, and levels of IFN- $\gamma$. Cat $\mathrm{S}^{-/-} L D L R^{-/-}$monocytes showed impaired subendothelial basement membrane transmigration, and aortas from $\mathrm{CatS}^{-/-} L D L R^{-/}$ mice had preserved elastic laminae. These findings establish a pivotal role for $\mathrm{Cat} S$ in atherogenesis.

J. Clin. Invest. 111:897-906 (2003). doi:10.1172/JCI200314915.

\section{Introduction}

ECM remodeling contributes to all stages of atherosclerosis. Proteolysis of ECM molecules probably participates in the initial mononuclear leukocyte translocation from the vascular lumen through the basement membrane into subendothelium, traversal of medial smooth muscle cells (SMCs) through the elastic laminae into the intima, compensatory arterial enlargement, plaque angiogenesis, and plaque disruption (1-3).

Previous studies have suggested involvement of both serine proteases and MMPs in arterial diseases. Deficiency of plasminogen reduced the development of lesions in transplanted mouse arteries (4) and in arteries of electrically injured mice (5). Hyperlipidemic mice deficient in urokinase showed reduced aortic ectasia (6), an effect attributed to reduced plasmin-dependent activation of pro-MMPs (6).

Human atheromata overexpress the interstitial collagenases MMP-1 (7), MMP-13 (8), and MMP-8 (9) at sites

Received for publication December 20, 2001, and accepted in revised form January 28, 2003.

Address correspondence to: Guo-Ping Shi, Surge 203, Box 0911, 90 Medical Center Way, San Francisco, California 94143, USA. Phone: (415) 514-1208; Fax: (415) 476-9749; E-mail: gpshi@itsa.ucsf.edu.

Galina K. Sukhova and Yaou Zhang contributed equally to this work.

Conflict of interest: The authors have declared that no conflict of interest exists.

Nonstandard abbreviations used: smooth muscle cell (SMC); cathepsin S (Cat S); endothelial cell (EC); monocyte chemoattractant protein-1 (MCP-1). of interstitial collagen cleavage. MMPs 2, 3, 7, 9, 12, 14, and others also localize in human atherosclerotic plaques $(7,10-14)$. Overexpression of the MMP inhibitor TIMP-1 reduced formation of aneurysms (15) and atherosclerotic lesions (16) in animals, affirming a role for MMPs in arterial remodeling and atherogenesis.

Beyond serine proteases and MMPs, we recently proposed the participation of cathepsin S (Cat S) and Cat K in atherogenesis. These cysteine proteases have potent elastolytic $(17,18)$ and collagenolytic activities $(19)$. Diseased human arteries overexpress these enzymes and exhibit a reciprocal deficiency of cystatin $\mathrm{C}$, the most abundant endogenous inhibitor of cysteine proteases $(1,2)$. Biochemical studies suggest that Cat $\mathrm{S}$ and $\mathrm{Cat} \mathrm{K}$ account for most of the elastolytic activity extracted from human carotid plaques. Multiple cell types found in human atheroma can express Cat S. We have previously demonstrated that intimal macrophages as well as SMCs in the tunica media express Cat $S$ in human atherosclerotic arteries (1). We also demonstrated colocalization of Cat $\mathrm{S}$ with lumenal and microvascular endothelial cells (ECs) in human atheroma and with microvascular ECs in wounded mouse skin (G.-P. Shi et al., unpublished data). In vitro experiments showed secretion of active Cat $\mathrm{S}$ by these atheroma-associated cell types following exposure to inflammatory cytokines found in atheroma. Our findings implicate Cat $\mathrm{S}$ in atherogenesis along with serine proteases and MMPs. Furthermore, Cat $S$, an essential protease acquired for invariant chain processing, plays an important role in antigen presentation. Cat $\mathrm{S}$ deficiency leads to impaired 
immune responses $(20,21)$, which might also affect atherogenesis $(22,23)$. Despite this plethora of observational and biochemical data, no direct evidence establishes a role of Cat $S$ in atherogenesis. This study used Cat Sdeficient $\left(\mathrm{CatS}^{-/-}\right)$mice crossbred with atherosclerosisprone $\left(L D L R^{-/}\right)$mice to address this question directly.

\section{Methods}

$\mathrm{CatS}^{-/-} \mathrm{LDLR}^{-/-}$mouse generation and animal protocol. $\mathrm{CatS}^{-/-}$mice were backcrossed more than ten generations onto the C57/BL6 background. These mice were crossbred with $L^{2} L R^{-/-}$mice (also on the C57/BL6 background; The Jackson Laboratory, Bar Harbor, Maine, USA). The resulting double knockout mouse genotypes were confirmed using mouse tail genomic DNA-derived PCR as described $(20,24)$. This study used both $\mathrm{CatS}^{-/-} \mathrm{LDLR}^{-/-}$and control $\mathrm{LDLR}^{-/-}$mice. Mice consumed an atherogenic diet (D12108; Research Diets Inc., New Brunswick, New Jersey, USA) containing $20.1 \%$ saturated fat, $1.37 \%$ cholesterol, and $0 \%$ sodium cholate (25). After 8, 12, and 26 weeks $(n=12$ / timepoint/group), mice were used as described below.

Oil red O staining for lipids. Deposition of lipids in enface preparations of abdominal aortas (fixed with $10 \%$ formalin) was determined by oil red O staining. Subsequently, the aortas were opened longitudinally to the iliac bifurcation and pinned out on the surface of black wax with $0.2-\mathrm{mm}$ steel pins. They were then stained with oil red $\mathrm{O}$ solution for 2.5 hours at room temperature on a shaker and washed three to four times in $85 \%$ propylene glycol solution.

Immunohistochemistry. Serial cryostat sections ( $6 \mu \mathrm{m})$ of mouse aortic arches were fixed in acetone, air dried, and stained by the avidin-biotin-peroxidase method. After limiting endogenous peroxidase activity with $0.3 \% \mathrm{H}_{2} \mathrm{O}_{2}$ and nonspecific binding of primary antibody with $5 \%$ species-appropriate normal serum (Vector Laboratories, Burlingame, California, USA), we incubated sections with primary antibodies diluted in PBS supplemented with 5\% species-appropriate normal serum for 90 minutes at room temperature. Incubation with secondary antibodies for 45 minutes was followed by avidin-biotin complex (ABC; Vector Laboratories) for 30 minutes. The reaction was visualized with 3-amino-9-ethylcarbazole (ready-to-use AEC; DAKO Corp., Carpinteria, California, USA) followed by counterstaining with Gill's hematoxylin solution (Sigma-Aldrich, St. Louis, Missouri, USA). Antibodies used were: rabbit anti-mouse Cat S (1:90; ref. 20), goat anti-smooth muscle $\alpha$-actin (1:75; Santa Cruz Biotechnology Inc., Santa Cruz, California, USA), rat anti-mouse macrophage (Mac-3, 1:1,000; Pharmingen, San Diego, California, USA), and rat anti-mouse $\mathrm{T}$ cell (CD4, 1:100; Pharmingen), goat anti-rat IFN- $\gamma$ (1:75; R\&D Systems Inc., Minneapolis, Minnesota, USA), hamster anti-mouse monocyte chemoattractant protein-1 (MCP-1) (1:50; Pharmingen), biotinylated anti-mouse VCAM-1 (1:60), and biotinylated anti-mouse ICAM-1 (1:50; Pharmingen).
Verboeff-van Gieson staining for elastin. This procedure used the Accustain Elastic Stain kit (Sigma-Aldrich) according to the manufacturer's recommendations.

Picrosirius red staining for type I and type III collagen. Formalin-fixed frozen sections were incubated for 4 hours in a freshly prepared $0.1 \%$ solution of Sirius Red F3BA (Polysciences Inc., Warrington, Pennsylvania, USA) in saturated aqueous picric acid. After rinsing twice in $0.01 \mathrm{~N} \mathrm{HCl}$ and distilled water, sections were briefly dehydrated and mounted in Permount (Vector Laboratories). Sirius red staining was analyzed by polarization microscopy.

Mouse aortic SMC isolation and elastase assay. Mouse aortic SMCs were isolated as described with modifications (26). Briefly, mouse aortas were removed and separated from fat and adventitia followed by digestion with $1 \times$ collagenase type II (Worthington Biochemical Corp., Lakewood, New Jersey, USA) at $37^{\circ} \mathrm{C}$ for $10-15$ minutes. The adventitia was completely stripped under microscopic visualization, the aortas were opened, and ECs were removed by abrasion. The aortas were then chopped and digested in $4 \mathrm{ml}$ of type I collagenase (1 $\mathrm{mg} / \mathrm{ml}$; Worthington Biochemical Corp.) and elastase III $(0.125 \mathrm{mg} / \mathrm{ml}$; Sigma-Aldrich) for $40-90$ minutes at $37^{\circ} \mathrm{C}$. Digestion was stopped by addition of $10 \mathrm{ml}$ of DMEM containing $10 \%$ FCS. Cells were then plated on a fibronectin-coated $60-\mathrm{mm}$ culture dish. After the first two passages, cells were cultured on gelatin-coated culture dishes. Cell identity was verified by immunostaining for $\alpha$-smooth muscle actin and calponin. To assay SMC elastase activity, SMCs were passaged onto 24-well plates $\left(1 \times 10^{5} /\right.$ well $)$. After the cells reached confluence, $300 \mu \mathrm{g}$ of $\left[{ }^{3} \mathrm{H}\right]$ elastin was added to each well followed by 24-48 hours of incubation in the presence or absence of IFN- $\gamma(500 \mathrm{U} / \mathrm{ml})$. Culture medium was collected by centrifugation $(14,000 \mathrm{~g}$ for 15 minutes) and soluble radioactive elastin was quantified as described (1).

Statistical analysis. To measure plaque size as well as the percent-positive area for staining, longitudinal sections of the aortic arch were analyzed microscopically for all mice. As described previously (27), a 3-mm segment of the lesser curvature of the aortic arch was defined proximally by a perpendicular axis dropped from the right side of the innominate artery. The percentage of the total area of the aortic arch stained for SMCs, macrophages, collagen, and lipids was determined using computerassisted image analysis (Image-Pro Plus; Media Cybernetics, Silver Springers, Maryland, USA).

We estimated atherosclerosis as well as immunohistochemical staining for MCP-1, VCAM-1, and ICAM-1 by semiquantitative grading using the following criteria: 0 , no staining; 1 , weak staining; 2 , moderate staining; 3, strong staining. Atherosclerotic lesions were graded as: 0 , no lesion; 1 , few macrophages underlying endothelium; 2, fatty streak-like lesion; and three grades ( 3 through 5) of atheroma, with the lower grades corresponding to less-developed lesions. Scoring was performed independently by two blinded observers. Data for $\mathrm{CatS}^{-/-} \mathrm{LDLR} \mathrm{R}^{-/-}$and control $L D L R^{-/-}$groups 
were presented as mean $\pm \mathrm{SD}$ and compared by the nonparametric Mann-Whitney test. A value of $P \leq 0.05$ was considered significant.

Lipoprotein measurement. Blood samples were collected by retro-orbital venous plexus puncture. Serum total cholesterol and triglyceride were assayed by enzymatic methods using Sigma Diagnostics kit nos. 401 and 343 (Sigma-Aldrich), respectively, adapted for microtiter plate assay. HDL was separated from LDL and VLDL cholesterol with kit no. 352-4, also adapted for microtiter plate assay. HDL cholesterol levels were then measured using kit no. 401. LDL cholesterol was calculated as follows: serum LDL cholesterol concentration $(\mathrm{mg} / \mathrm{dl})=$ total cholesterol - HDL cholesterol - (triglycerides/5).

Mouse monocyte isolation, transmigration measurement, and invasion. Monocytes from $\mathrm{CatS}^{-/-} \mathrm{LDLR}^{-{ }^{-}}$and $L D L R^{-/-}$mice were isolated by Percoll gradient centrifugation. Briefly, mouse blood was collected into heparin (1:200; Sigma-Aldrich) followed by 1:1 dilution with $1 \times$ PBS. Diluted blood was laid over a Percoll gradient $(1.04 \mathrm{~g} / \mathrm{ml}$; Amersham Biosciences Corp., Piscataway, New Jersey, USA) and centrifuged for 30 minutes at $900 \mathrm{~g}$. The interface layer of monocytes/lymphocytes was collected and cultured on a Petri dish for 2 hours. Unattached lymphocytes were removed by washing with RPMI and monocytes were further cultured in RPMI.

Rabbit aortic SMCs and ECs were cocultured as described (28). Briefly, $1 \times 10^{6}$ rabbit aortic SMCs were cultivated with EGM- 2 media (containing $2 \%$ rabbit plasma) on the filter for more than 3 weeks. The mixture of type I and type IV collagen was then laid on the filter of a Chemotaxicell chamber (Kurabo Inc., Osaka, Japan) and incubated at $37^{\circ} \mathrm{C}$ for 1 hour to complete gel formation. Then $2 \times 10^{5}$ rabbit aortic EC's were seeded on top of the gel; these became confluent in a week. Monocytes $\left(1 \times 10^{6}\right)$ isolated from peripheral blood of $\mathrm{CatS}^{-/-} L D L R^{-/-}$or $L D L R^{-/-}$mice were applied to the EC layer (day 0). After 7 days of culture, samples were fixed with $10 \%$ formalin, embedded in paraffin, and sectioned $(10 \mu \mathrm{m})$ for immunohistochemical study. Sections were incubated with primary antibodies: F4/80 (anti-mouse monocyte, ref. 29) or anti-macrophage scavenger receptor class A, type I (anti-MSR) (30) followed by secondary antibody conjugated with HRP (Amersham Biosciences Corp.). After visualization with 3,3'-diaminobenzidine (Dojin Chemical Co., Kumamoto, Japan), nuclei were counterstained with hematoxylin. The numbers of monocytes/macrophages attached to ECs and those that had transmigrated into the matrix layer were counted by two blinded observers using confocal microscopy (TCS SP; Leica Microsystems GmbH, Heidelberg, Germany) as described previously (28). Results are presented as mean \pm SEM. Statistical comparisons were made using the two-tailed Student paired $t$ test; a value of $P \leq 0.01$ was considered significant. The experiment was repeated four times.

For the mouse monocyte invasion/transmigration assay, cells were incubated overnight at room temperature in PBS in Boyden transwell chambers coated with type I collagen $(110 \mu \mathrm{g} / \mathrm{ml}$; Celtrix Pharmaceuticals Inc., Santa Clara, California, USA) and type IV collagen $(30 \mu \mathrm{g} / \mathrm{ml}$; BD Biosciences, Bedford, Massachusetts, USA). Briefly, monocytes were resuspended in serumfree media at $1 \times 10^{5} / \mathrm{ml}$, and $0.5 \mathrm{ml}$ of suspension was added to each well. The lower chamber was supplied with $0.8 \mathrm{ml}$ of complete media with $0.5 \mu \mathrm{g}$ of mouse recombinant MCP-1 (Pharmingen). The invasion assay was carried out at $37^{\circ} \mathrm{C}$ for 16 hours followed by crystal violet staining. Six microscopic fields were randomly chosen to count transmigrated cells.

\section{Table 1}

Plasma lipid contents in mice fed with chow or high cholesterol diet

\begin{tabular}{|c|c|c|c|c|c|c|}
\hline Genotype & Diet & $n$ & $\begin{array}{l}\text { Plasma total } \\
\text { cholesterol } \\
(\mathrm{mg} / \mathrm{dl})\end{array}$ & $\begin{array}{c}\text { Plasma total } \\
\text { triglyceride } \\
(\mathrm{mg} / \mathrm{dl})\end{array}$ & $\begin{array}{c}\text { Plasma total } \\
\text { HDL } \\
(\mathrm{mg} / \mathrm{dl})\end{array}$ & $\begin{array}{c}\text { Plasma total } \\
\text { LDL } \\
(\mathrm{mg} / \mathrm{dl})\end{array}$ \\
\hline WT & & 10 & $169.7 \pm 49.7$ & $104.6 \pm 32.7$ & $57.4 \pm 4.5$ & $91.4 \pm 40.1$ \\
\hline $\mathrm{CatS}^{-/-}$ & & 12 & $171.5 \pm 50.0$ & $110.3 \pm 42.3$ & $55.6 \pm 5.6$ & $93.9 \pm 24.0$ \\
\hline$L D L R^{-/-}$ & Chow & 6 & $291.1 \pm 44.7$ & $112.8 \pm 26.8$ & $46.3 \pm 18.7$ & $222.3 \pm 52.4$ \\
\hline $\mathrm{CatS}^{-/-} / L D L R^{-/-}$ & & 6 & $237.2 \pm 28.2^{\mathrm{A}}$ & $116.3 \pm 28.0$ & $49.6 \pm 18.8$ & $164.4 \pm 23.4^{\mathrm{A}}$ \\
\hline WT & & 8 & $200.1 \pm 43.3$ & $115.2 \pm 28.7$ & $52.4 \pm 8.9$ & $124.6 \pm 33.3$ \\
\hline $\mathrm{CatS}^{-/-}$ & & 8 & $215.2 \pm 31.3$ & $120.1 \pm 25.3$ & $62.3 \pm 15.9$ & $129.0 \pm 25.7$ \\
\hline$L D L R^{-/-}$ & High cholesterol & 6 & $1,519.7 \pm 124.0$ & $207.2 \pm 38.1$ & $49.1 \pm 27.9$ & $1,429.1 \pm 149.8$ \\
\hline $\mathrm{CatS}^{-/-} / L D L R^{-/-}$ & (8 weeks) & 8 & $1,348.8 \pm 236.9$ & $162.8 \pm 82.6$ & $50.4 \pm 22.7$ & $1,265.8 \pm 233.9$ \\
\hline WT & & 8 & $221.6 \pm 52.7$ & $118.0 \pm 23.7$ & $55.5 \pm 8.7$ & $142.5 \pm 30.0$ \\
\hline $\mathrm{CatS}^{-/-}$ & & 8 & $235.1 \pm 30.5$ & $112.7 \pm 24.2$ & $57.6 \pm 28.3$ & $155.0 \pm 33.1$ \\
\hline$L D L R^{-/-}$ & High cholesterol & 12 & $1,355.2 \pm 340.1$ & $190.9 \pm 39.7$ & $64.2 \pm 9.3$ & $1,251.7 \pm 343.4$ \\
\hline $\mathrm{CatS}^{-/-} / L D L R^{-/-}$ & (12 weeks) & 12 & $1,214.4 \pm 295.3$ & $204.8 \pm 60.3$ & $70.2 \pm 15.0$ & $1,103.2 \pm 396.2$ \\
\hline WT & & 7 & $195.6 \pm 35.7$ & $121.5 \pm 28.8$ & $58.7 \pm 13.2$ & $112.5 \pm 32.4$ \\
\hline $\mathrm{CatS}^{-/-}$ & & 7 & $180.5 \pm 43.2$ & $119.1 \pm 31.5$ & $62.0 \pm 22.7$ & $94.7 \pm 24.2$ \\
\hline$L D L R^{-/-}$ & High cholesterol & 11 & $851.7 \pm 162.1$ & NA & $54.1 \pm 5.0$ & $770.4 \pm 198.8$ \\
\hline $\mathrm{CatS}^{-/-} / L D L R^{-/-}$ & (26 weeks) & 11 & $949.1 \pm 210.9$ & NA & $57.8 \pm 8.6$ & $862.8 \pm 170.5$ \\
\hline
\end{tabular}

AP $<0.04$, CatS $^{-/-} / L D L R^{-/-}$mice fed with chow diet vs. $L D L R^{-/-}$mice fed with chow diet. NA, not assayed. 


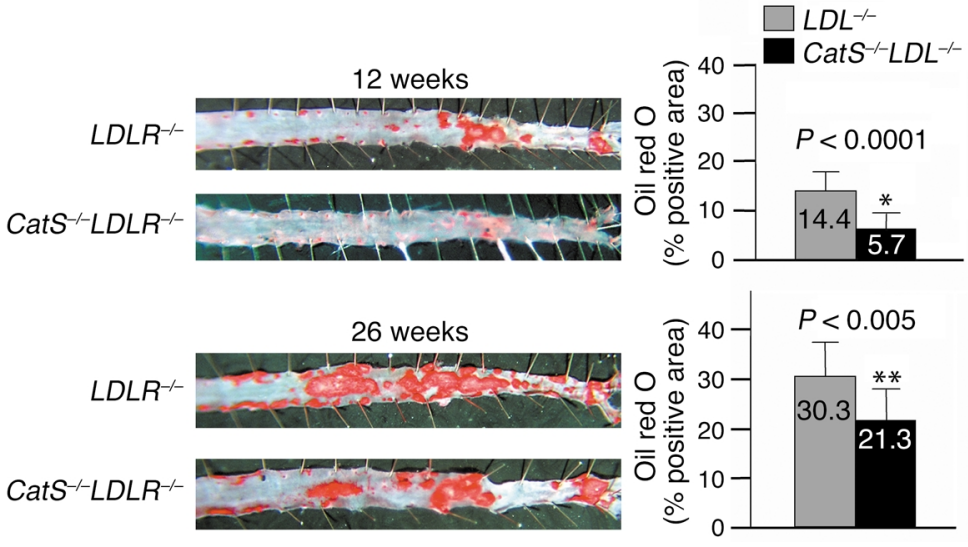

Figure 1

Cat $\mathrm{S}$ deficiency reduces the area of aortic lipid accumulation. After consuming the atherogenic diet for 12 or 26 weeks, both $\mathrm{CatS}^{-/-} L D L R^{-/-}$and $L D L R^{-/-}$mouse abdominal aortas were isolated and stained for lipid deposition with oil red $\mathrm{O}$. The left panels show representative specimens used to calculate the percentages of oil red $\mathrm{O}$-positive areas shown in the bar graphs on the right. No aortic lipid deposition was seen in mice fed an atherogenic diet for 8 weeks (data not shown).

Cat $S$ deficiency does not affect serum lipids. In agreement with earlier findings, LDLR deficiency significantly increased plasma total cholesterol and LDL levels but did not change either HDL or triglyceride levels (24). Disruption of CatS gene function alone did not affect plasma total cholesterol, triglyceride, HDL, or LDL levels in mice consuming an atherogenic diet (Table 1). $\mathrm{Cat}^{-/-} L D L R^{-/-}$mice that consumed either a chow or atherogenic diet showed no significant differences in plasma total cholesterol and total LDL levels compared with similarly fed $L D L R^{-/-}$mice (Table 1 ).

Cat $S$ deficiency reduces atherosclerosis in $L D L R^{-/-}$mice. $L D L R^{-/-}$mice fed an atherogenic diet developed atherosclerotic lesions with morphological characteristics similar in many aspects to those seen in humans (31). To evaluate the severity of atherosclerosis in mice, we characterized lesions by (a) lesion area in the abdominal aorta delineated by lipid staining with oil red $\mathrm{O}$ (Fig-

\section{Results}

Generation of $\mathrm{CatS}^{-/-} L D L R^{-/-}$mice. $L D L R^{-/-}$mice develop atherosclerotic plaques when consuming a highcholesterol diet and provide a well-established model of atherosclerosis (31).

To study the role of Cat $\mathrm{S}$ in atherogenesis, we generated double-knockout ( $\mathrm{CatS}^{-/-} L D L R^{-/-}$) mice by crossing $\mathrm{CatS}^{-/-}$mice with $L D L R^{-/-}$mice, both on the C57/BL6 background. PCR analysis of tail genomic DNA confirmed the presence of mutant alleles for both Cat $S$ (170 bp) (20) and LDLR (800 bp) (24), as well as the absence of their corresponding wild-type alleles (data not shown). Both $\mathrm{CatS}^{-/-}$and $\mathrm{CatS}^{-/-} L D L R^{-/-}$mice were apparently healthy and fertile, and exhibited mendelian transmission of the mutant alleles. We noticed no obvious abnormality in vasculature, organ development, gestation time, or food/water consumption. ure 1), (b) quantitative morphometric measurement of intimal thickening (area between internal elastic lamina and lumen) in longitudinal sections of aortic arches (intimal area; Figure 2a), and (c) histological grading of lesions in aortic arches (see details in Methods) (Figure 2b). Analysis of en-face preparations of abdominal aorta stained with oil red $\mathrm{O}$ showed a statistically significant reduction of plaque area in $\mathrm{CatS}^{-/-} L D L R^{-/-}$mice after 12 weeks ( $60 \%$ reduction, $P<0.0001)$ and 26 weeks $(30 \%$ reduction, $P<0.005$ ) on an atherogenic diet (Figure 1 ). Consistent with the observations on the abdominal aorta, the aortic arches from $\mathrm{CatS}^{-/-} L D L R^{-/-}$mice also showed a statistically significant reduction in plaque size (intimal area; Figure 2a) as well as stage of plaque development (Figure $2 \mathrm{~b}$ ) compared with $L D L R^{-/-}$mice at all three timepoints tested. In comparison, Cat $S$ single-deficient mice consuming the high-cholesterol diet $\mathbf{a}$

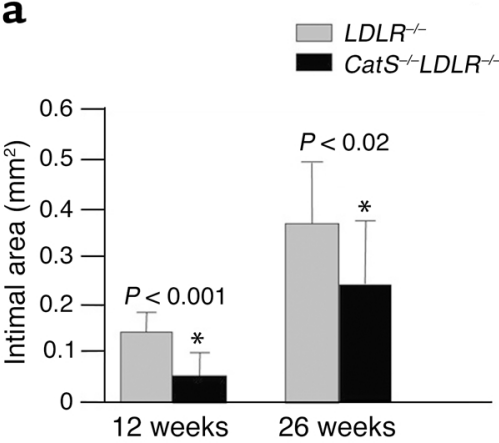

b

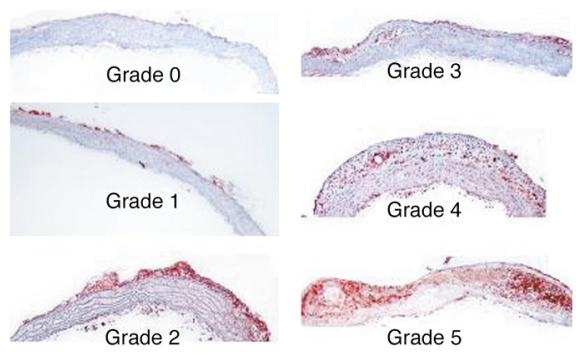

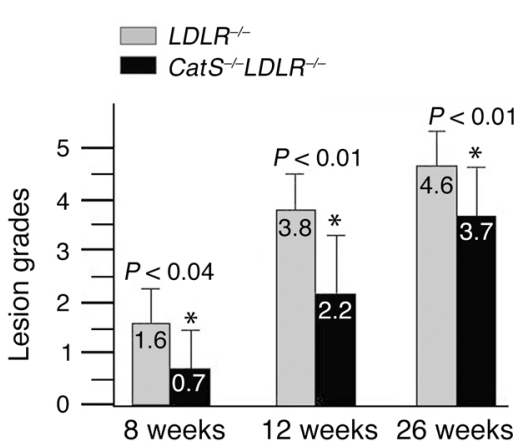

Figure 2

Attenuated aortic arch atherosclerotic lesions in $\mathrm{CatS}^{-/-} L D L R^{-/-}$mice. Aortic arches were sectioned and stained with anti-Mac-3 antibodies as described in Methods. (a) Measurement of aortic arch intimal area. Neointimal area $\left(\mathrm{mm}^{2}\right)$ was analyzed in 3-mm longitudinal sections of the aortic arches. CatS $S^{-1-} L D L R^{-/-}$mice showed statistically significant reductions in intimal areas at both the 12-week and 26-week timepoints compared with $L D L R^{-/-}$mice. (b) Illustration of atherosclerotic lesion grading. Each group of mice $(n=12)$ consumed an atherogenic diet. Lesions were graded as displayed in the left panels. Cat S deficiency significantly reduced atherosclerotic lesions at 8,12 , and 26 weeks (right panel). 


\section{Figure 3}

Preserved elastin in $\mathrm{CatS}^{-/-} L D L R^{-/-}$mice. (a) Verhoeff-van Gieson staining for elastin shows aortic arch elastic laminae. The graph shows the number of discontinuities in the elastic lamina enumerated in the 3-mm aortic arch sections. Significant differences in elastin preservation between $\mathrm{CatS}^{-/-} L D L R^{-/-}$and $L D L R^{-/-}$mice occurred at both the 12-week and 26-week timepoints. (b) Cat Spositive medial SMCs colocalized with sites of elastin degradation in $L D L R^{-/-}$mice. Serial sections of aortic arches were stained for $\alpha$-actin (top left) and Cat S (bottom left). Verhoeff-van Gieson staining for elastin performed after $\mathrm{Cat} \mathrm{S}$ staining showed $\mathrm{Cat} \mathrm{S}$ expression to be colocalized with elastin fragmentation; arrows indicate elastin breaks (right). (c) SMC elastase assay. IFN- $\gamma$-stimulated SMCs from $\mathrm{CatS}^{-1-}$ mouse aortas showed significantly weaker elastase activity than was found in wild-type cells $(P<0.0001)$. Three independent experiments yielded similar observations. (d and $\mathbf{e}$ ) Reduction of intimal SMCs, collagen content, and fibrous cap thickness in $\mathrm{CatS}^{-1-} L D L R^{-1-}$ mice. Longitudinal sections of aortic arch were immunostained for $\alpha$-actin (SMC content) and for collagen content with picrosirius red. $\mathrm{CatS}^{-/-} L D L R^{-/-}$mice had significantly less SMC and collagen in intima than did $L D L R^{-/-}$mice. Percentages of positively stained areas were calculated using computer-assisted image quantification as described (27). Values represent mean $\pm \mathrm{SD}, P$ values $\leq 0.05$ were considered significant. (f) The thickness of the fibrous cap of an aortic atheroma of each mouse was measured using sections stained with oil red $\mathrm{O}$ as described in Methods. Few if any fibrous caps formed at earlier timepoints.

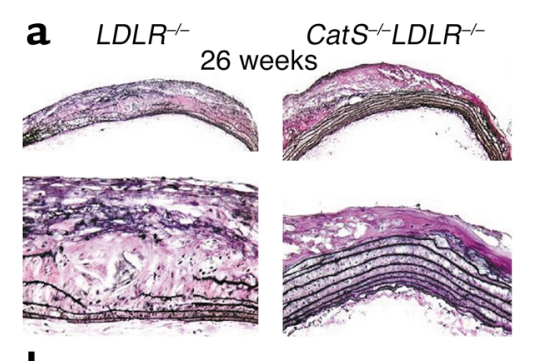

b
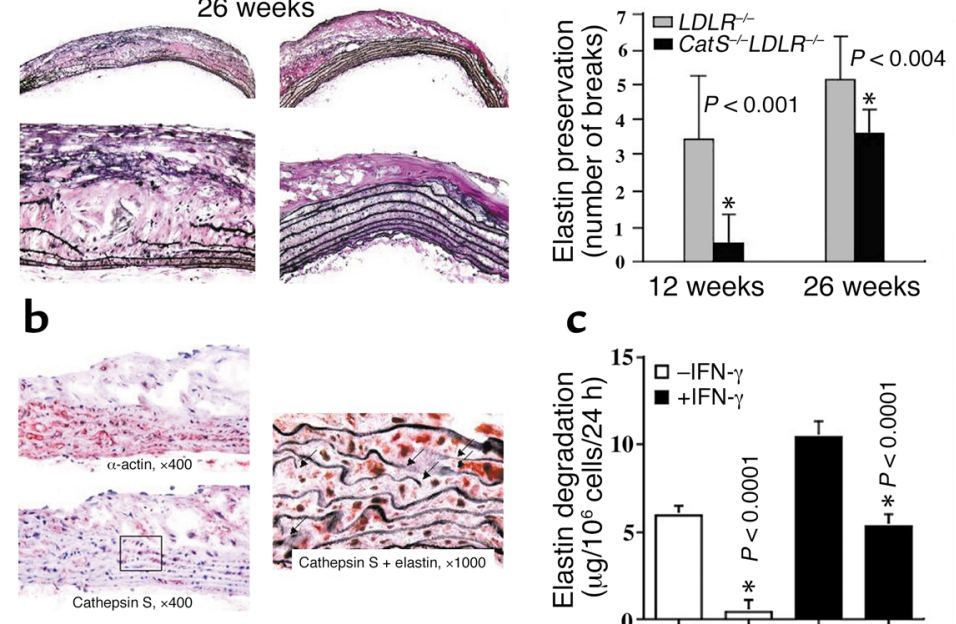

C
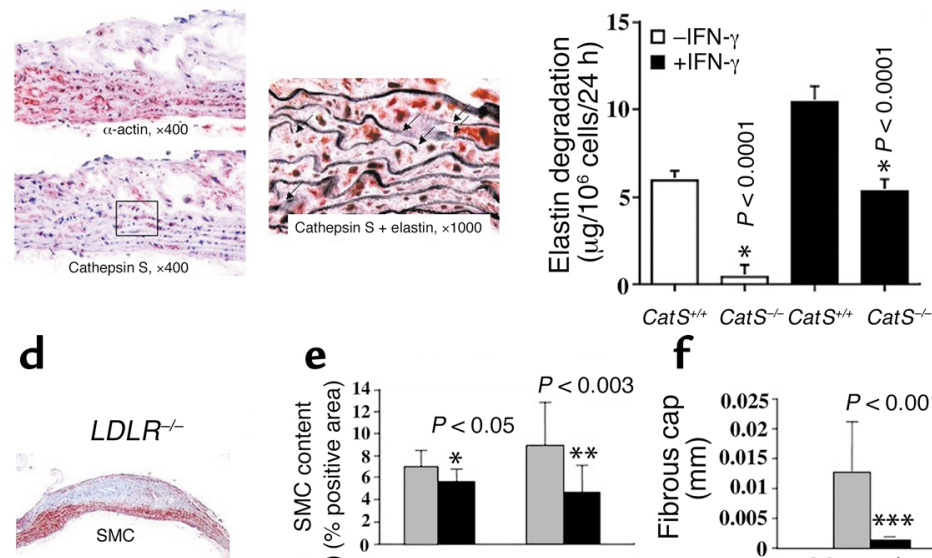

e

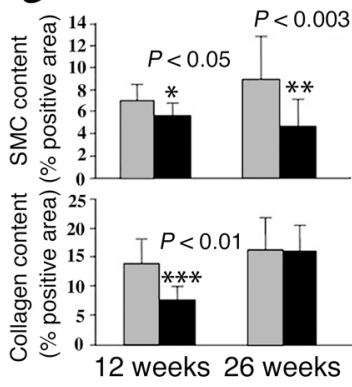

$f$
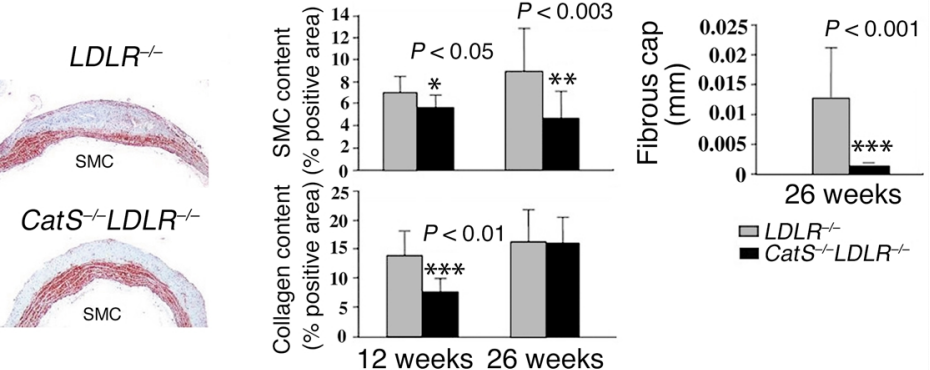

(free of cholate) did not develop any lesions at any timepoint tested (data not shown).

Elastin preservation in $\mathrm{CatS}^{-/-} L D L R^{-/-}$mice. The arterial ECM contains abundant elastin. Degradation (fragmentation) of elastic laminae contributes to SMC penetration into the intima during the formation of atheroma, compensatory enlargement, and in the extreme, development of atherosclerotic aneurysms. Elastic laminae in the aortic arch showed preserved integrity in $C a t S^{-/-} L D L R^{-/}$mice compared with those wild type for Cat $S$, a potent elastase (Figure 3a). The doubly-deficient mice showed significantly fewer elastin breaks at both 12 weeks $(P<0.001)$ and 26 weeks $(P<0.004)$ after initiation of the atherogenic diet (Figure 3a). Importantly, as in human atheromata (1), Cat $S$ colocalizes with $\alpha$-actinpositive SMCs in the media underlying atherosclerotic plaques in $L D L^{-/-}$mice (Figure $3 b$ ). Furthermore, Verhoeff-van Gieson staining for elastin on Cat S-stained sections revealed colocalization of Cat S-positive SMCs with breaks in the elastic laminae (Figure $3 \mathrm{~b}$, right), supporting a role of Cat $\mathrm{S}$ in elastolysis in situ. To examine whether Cat $\mathrm{S}$ from SMCs can degrade extracellular elastin in vitro, we isolated SMCs from both $\mathrm{CatS}^{-/-}$and control mice and measured their elastolytic capacity. SMCs from $\mathrm{CatS}^{-/-}$mice have much less elastase activity even when stimulated with IFN- $\gamma(P<0.0001)$ (Figure $3 c)$.
Reduced SMC and interstitial collagen content of intimal lesions in $\mathrm{CatS}^{-/-} L D L R^{-/-}$mice. SMC migration from the tunica media into the intima requires proteolytic degradation of elastin. The early lesions in aortas of $\mathrm{CatS}^{-/-} L D L R^{-/-}$mice contained significantly fewer SMCs than those in $L D L R^{-/-}$mice, measured as the percent of $\alpha$-actin-positive area at 12 weeks $(P<0.05)$ and 26 weeks $(P<0.003)$ (Figure 3, d and e). Picrosirius red staining of the aortic arches revealed a significant reduction in collagen content in $\mathrm{CatS}^{-/-} \mathrm{LDLR}^{-/-}$ mice compared with $L D L R^{-/-}$control mice at the 12week timepoint $(P<0.01)$ but not at the 26 -week timepoint (Figure 3e). Atheromata in $\mathrm{CatS}^{-/-} L D L R^{-/-}$mice had significantly thinner fibrous caps at 26 weeks (Figure $3, \mathrm{~d}$ and $\mathrm{f}, P<0.001$ ). The fibrous caps were insufficiently developed at earlier timepoints to permit accurate measurement. Reduction of both the collagen content and the fibrous cap thickness may reflect the decreased number of SMCs (the major source of collagen in arteries) due to impaired SMC migration across the internal elastic membrane that separates the tunica media from the intima.

Cat $S$ deficiency decreases macrophage and leukocyte accumulation in aortic lesions. Macrophages sustain inflammation within atheromata and probably influence plaque stability. Human atheromata that have ruptured and caused fatal thrombosis typically have abundant macrophages (32). The percentage of plaque area 
a

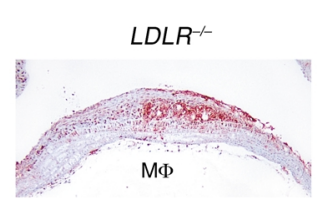

Cat $^{-1-} L D L R^{-1}$

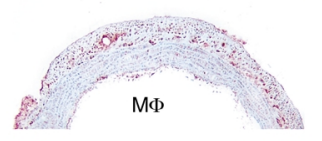

d

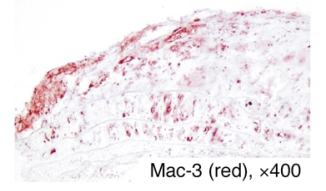

b
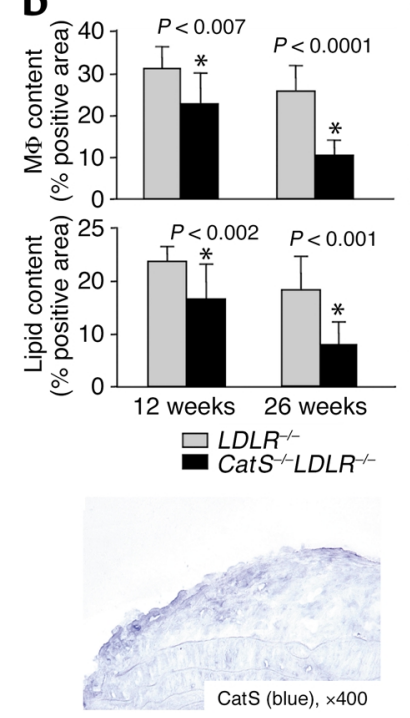

C
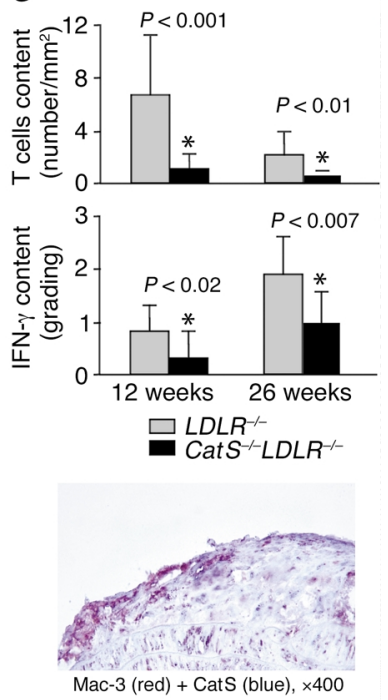

Figure 4

Cat $\mathrm{S}$ deficiency changes the composition of atheromata. Longitudinal sections of the aortic arch were immunostained for Mac-3 (macrophages) (a), oil red O (lipids), CD4 (T lymphocytes) or IFN- $\gamma$. (b and $\mathbf{c}$ ) Computer-assisted image analysis quantified the percentage of positively stained areas (27). Values represent mean \pm SD; $P$ values $\leq 0.05$ were considered significant. (d) Double immunostaining for Cat $S$ (blue, middle) and macrophages (Mac-3, red, left) showed colocalization of these two antigens (purple, right). Analysis of four specimens showed similar results. $\mathrm{M} \phi$, macrophage.

composed of Mac-3-positive monocytes/macrophages decreased significantly in $\mathrm{CatS}^{-/-} L D L R^{-/-}$mice at both the 12 -week $(P<0.007)$ and 26 -week $(P<0.0001)$ timepoints compared with $L D L R^{-/-}$controls (Figure 4 , a and $\mathrm{b}$, upper panels). Oil red $\mathrm{O}$ staining for lipid accumulation in the aortic arch declined in parallel with the content of macrophages, the major repository of intracellular lipid in plaques, at both timepoints $(P<0.002$ and $P<0.001$ ) (Figure $4 \mathrm{~b}$, bottom). Similar to macrophages in human atheromata (1), Mac-3-positive macrophages (Figure 4d, red) express Cat S (Figure 4d, blue) within intimal lesions in $L D L R^{-/-}$mice. These two antigens colocalize, as shown by double immunostaining on the same section (Figure 4d, purple).

The lenkocytic infiltrates in atheroma include lymphocytes and mononuclear phagocytes. $\mathrm{CD} 4^{+} \mathrm{T}$ cells, the predominant lymphocytes found in human and murine atherosclerotic plaques, participate in the chronic immune response characteristic of atherosclerosis $(33,34)$. We therefore enumerated $\mathrm{CD}^{+} \mathrm{T}$ cells in aortic arches. Lesions from $\mathrm{CatS}^{-/-} L D L R^{-/-}$mice had significantly fewer $\mathrm{CD} 4^{+} \mathrm{T}$ cells than did $L D L R^{-/}$controls (Figure $4 c$, top). These changes in lymphocyte accumulation did not merely reflect the decreased lesion size in the $\mathrm{CatS}^{-/-} L D L R^{-/-}$mice, as their content was normalized to lesion area. Consistent with the reduction of $\mathrm{CD}^{+}$ $\mathrm{T}$ cells in the $\mathrm{CatS}^{-/-} L D L R^{-/-}$mouse aorta, the amount of the prototypic Th1 cytokine, IFN- $\gamma$, also diminished significantly compared with its levels in $L D L R^{-/-}$mice (Figure 4c, bottom). Similar to macrophages, $\mathrm{CD}^{+}$ $\mathrm{T}$ cells, though fewer in atheromata, also express immunoreactive Cat S (data not shown), suggesting that Cat $\mathrm{S}$ might also influence $\mathrm{T}$ lymphocyte trafficking during atherogenesis.

Cat $S$ deficiency impairs leukocyte transmigration through subendothelial basement membrane. We first tested the possibility that reduced leukocyte accumulation in $\mathrm{CatS}^{-/-} \mathrm{LDLR}^{-/-}$mice resulted from decreased expression of adhesion and chemoattractant molecules. However, we detected no difference in the expression of VCAM-1, ICAM-1, or MCP-1 at the early timepoint (8 weeks) most important for white blood cell recruitment (Table 2) (35). After 12 weeks and 26 weeks on an atherogenic diet when atheromatous lesions were rather well developed, lesions did show a statistically significant decrease in immunoreactive VCAM-1 $(P<0.02$ for both the 12 -week and 26-week timepoints) or MCP- $1(P<0.004$ and $P<0.006$ for 12 -week and 26-week timepoints, respectively). In addition to reduced local inflammatory activation, these changes may reflect decreased atheroma formation (36). ICAM-1 protein content did not differ among groups at any time studied.

Alternatively, Cat S might participate in leukocyte migration from peripheral blood by facilitating proteolytic degradation of subendothelial vascular basement membrane. The nascent atheromata in $L D L R^{-/-}$mice (Figure 2, grade 1 and grade 2) had Cat S-positive macrophages underlying the endothelium (Figure 5a). We previously showed a similar pattern of Cat $\mathrm{S}$ expression in human fatty streaks (1). We tested this hypothesis in vitro in a monocyte migration and differentiation assay described previously (28). This assay measures migration of peripheral blood monocytes isolated from $\mathrm{CatS}^{-/-} L D L R^{-/-}$and control $L D L R^{-/-}$mice through a monolayer of ECs cultured atop a mixture of type I and type IV collagens, prominent components of the subendothelial basement membrane (see details in Methods). The formation of an EC monolayer as well as the deposition of a collagen I and collagen IV-containing basement membrane has been previously demonstrated (28). After 7 days of incubation, $91 \%$ of the $\mathrm{F} 4 / 80$-positive monocytes/macrophages (29) from $\mathrm{CatS}^{-/-} L D L R^{-/}$mice remained in the upper chamber, and only $9 \%$ of these cells transmigrated through the endothelial monolayer and the subjacent collagen-containing substrate (Figure $5, \mathrm{~d}$ and $\mathrm{f}$ ). In contrast, over $50 \%$ of $L D L R^{-/-}$monocytes/macrophages crossed into the SMC layer (Figure 5, 
Table 2

Grading of adhesion molecule expression in atherosclerotic lesions

\begin{tabular}{|c|c|c|c|c|}
\hline Western diet & Mouse genotype & MCP-1 & VCAM-1 & ICAM-1 \\
\hline \multirow[t]{3}{*}{8 weeks } & $L D L R^{-/-}$ & $0.79 \pm 0.88$ & $1.37 \pm 0.38$ & $1.75 \pm 0.69$ \\
\hline & $\mathrm{CatS}^{-/-} L D L R^{-/-}$ & $1.00 \pm 0.71$ & $1.00 \pm 0.77$ & $1.33 \pm 0.26$ \\
\hline & & NS & NS & NS \\
\hline \multirow[t]{3}{*}{12 weeks } & $L D L R^{-/-}$ & $2.33 \pm 0.61$ & $2.75 \pm 0.42$ & $1.50 \pm 0.71$ \\
\hline & $\mathrm{CatS}^{-/-} L D L R^{-/-}$ & $0.29 \pm 0.25$ & $1.96 \pm 0.56$ & $1.17 \pm 0.41$ \\
\hline & & $P<0.004$ & $P<0.02$ & NS \\
\hline \multirow[t]{3}{*}{26 weeks } & $L D L R^{-/-}$ & $2.25 \pm 0.69$ & $3.13 \pm 0.44$ & $0.37 \pm 0.34$ \\
\hline & $\mathrm{CatS}^{-/-}{ }^{-1} L R^{-/-}$ & $0.50 \pm 0.52$ & $2.37 \pm 0.38$ & $0.83 \pm 0.74$ \\
\hline & & $P<0.006$ & $P<0.02$ & NS \\
\hline
\end{tabular}

NS, no significant difference.

$\mathrm{c}$ and $\mathrm{f}, P<0.004)$. Immunostaining for macrophage scavenger receptor-A confirmed the identity of these cells as differentiated macrophages (Figure 5e, arrowheads). These observations suggest involvement of Cat $S$ in peripheral blood leukocyte transmigration through the vessel wall subendothelial basement membrane. This process may contribute to superficial erosion of atheroma, a cause of some fatal coronary thrombi, particularly in women and diabetics $(37,38)$.

To determine whether collagen-containing subendothelial basement membrane proteolysis by Cat $\mathrm{S}$ might contribute to blood-borne leukocyte transmigration, a simple cell invasion assay was performed using Boyden transwells coated with collagen types I and IV, using MCP-1 as chemoattractant. Although fewer $\mathrm{CatS}^{-/-}$cells (mean of six randomly chosen fields) transmigrated through the collagen layer than did control cells ( $58 \pm 22$ vs. $92 \pm 23$ ), this trend did not achieve significance $(P>0.05)$, arguing against a major role for Cat $\mathrm{S}$ in degrading collagen-containing basement membranes. This finding agrees with the weak collagenolytic activity of Cat S (39) and suggests operation of other mechanisms of inhibition of macrophage accumulation, perhaps related to the reduced levels of MCP-1 in atheromata of CatS $^{-/-} L_{D L R^{-/-}}$mice.

\section{Discussion}

Recent observations have implicated the elastolytic cathepsins $\mathrm{S}$ and $\mathrm{K}$ in atherosclerosis $(1,2)$. The present study sought direct evidence for Cat $\mathrm{S}$ involvement in atherogenesis using genetically modified mice. These experiments showed in vivo that lack of Cat $\mathrm{S}$ reduced atherogenesis in $L D L R^{-/-}$ mice by more than $50 \%$ after 8 or 12 weeks of consumption of an atherogenic diet, and mice still showed significantly diminished atheroma ( $\sim 30 \%$ reduction) at 26 weeks (Figure 1 and Figure 2). This attenuation of atherosclerosis in $\mathrm{CatS}^{-/-} L D L R^{-/-}$mice likely results primarily from the lack of Cat $\mathrm{S}$. We found no difference in other cysteine proteases (Cat B and Cat L, examined by cysteine protease active site [ $\left.{ }^{125} \mathrm{I}\right]-\mathrm{JPM}$ labeling) nor in the expression of MMPs (MMP-2, MMP-3, or MMP-9 examined by Western blot analysis) in $\mathrm{CatS}^{-/-} L D L R^{-/-}$compared with $L D L R^{-/-}$macrophages (data not shown). Nor did deficiency of Cat $S$ affect serum lipid levels or expression of other cathepsins or MMPs by ECs (G.-P. Shi et al., unpublished data). The striking magnitude and duration of this blunted lesion formation highlights the importance of understanding the mechanism(s) of Cat S involvement in atherogenesis.

Although not conclusive, our results suggest several possible mechanisms by which Cat $\mathrm{S}$ may contribute to lesion formation. First, Cat $S$, one of the most potent mammalian elastases (40), remains active at neutral $\mathrm{pH}$ (18) and may participate in proteolysis of elastic laminae in arteries $(1,2)$. We previously demonstrated in vitro that SMCs activated by IFN- $\gamma$ as well as differentiated macrophages can express and secrete active Cat $\mathrm{S}$ and degrade extracellular elastin. Either natural (cystatin C) or synthetic inhibitors of Cat $S$ can substantially block this elastolysis $(1,2,41,42)$. Within the artery wall, elastin fibers contribute to the vessel's structural integrity and regulate SMC migration and proliferation (43). Mice with targeted disruption of the elastin gene die 2-3 days after birth due to malformed blood vessels (44). Our present observations indicate that degradation or remodeling of elastic laminae contributes to generation
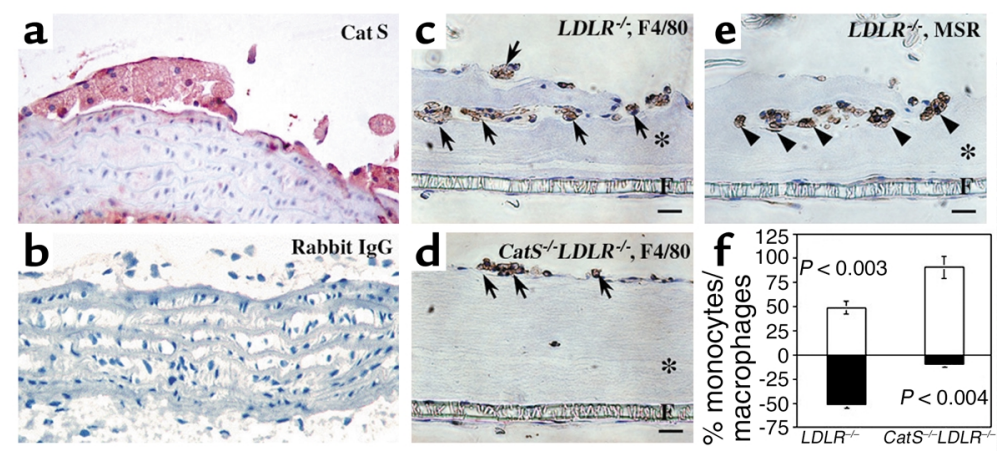

Figure 5

Cat $\mathrm{S}$ deficiency impairs leukocyte transmigration. (a) Increased local expression of Cat $S$ by ECs and macrophages abutting the arterial basement membrane. Paraffin sections of $L D L R^{-/-}$mouse fatty streaks were stained with rabbit anti-mouse Cat $S$ antibodies (red color) and non-immune rabbit IgG (b). (c-e) In vitro monocyte transmigration. Cat S deficiency inhibited transmigration of monocytes/macrophages through the EC monolayer and collagen-containing film prepared as described in Methods. Transmigrated macrophages were detected by the monocyte/macrophage marker F4/80 (arrows) (c and d) and macrophage scavenger receptor (MSR) antibodies (arrowheads) (e). Asterisks and Fs indicate collagen matrix and microporous filter, respectively. (f) The bar graph shows percentage of monocytes/macrophages remaining on the EC layer (white bars) and the percentage that transmigrated into the collagen-containing gel (black bars). Six independent experiments showed similar results. 
and development of atheromatous lesions, indicating a critical role for elastin preservation in this postnatally acquired disease as well. Indeed, in this study, Cat S had the greatest overall effect on lesion development at 12 weeks, when Cat $S^{-/-} L D L R^{-/-}$mice still showed better preserved elastic laminae in aortic arches (Figure 3a) and abdominal aortas (data not shown) than in $L D L R^{-/-}$mice. Similar to our earlier findings in human atheromata (1), medial SMCs in $L D L R^{-1-}$ mice also express abundant $\mathrm{Cat} S$ at the sites of elastic lamina degradation (Figure $3 b$ ), suggesting a role for Cat $S$ in this process. Primary cultured $\mathrm{CatS}^{-/}$SMCs stimulated with IFN- $\gamma$ showed significantly lower elastolysis than did SMCs from control mice (Figure 3c). Reduced elastin breakdown may limit SMC entry into the intima across the elastin barriers in $\mathrm{CatS}^{-/-} L D L R^{-/-}$mice. The thinner fibrous cap and reduced intimal collagen content may follow decreased SMC accumulation in lesions, as these cells produce most of the collagen in atherosclerotic lesions (45).

A second mechanism of Cat $\mathrm{S}$ function may involve remodeling of the basal lamina or basement membrane of the artery's intimal layer. Immunostaining for Cat $\mathrm{S}$ showed strong signals in the basement membrane regions of human fatty streaks (1). We observed a similar pattern of Cat $S$ expression in fatty streaks of $L D L R^{-/-}$mice (Figure $5 \mathrm{a}$ ). To enter the intima during early atherogenesis, leukocytes must penetrate the arterial basement membrane, which contains type IV and type I collagen, laminin, and fibronectin (46). This study monitored monocyte transmigration in vitro through layers of SMCs, collagen matrix, and ECs. Scanning EM analysis demonstrated monocyte traversal through both the EC monolayer and the underlying collagen matrix (28). The present experiments suggest that Cat $\mathrm{S}$ may pave the way for leukocyte entry by breaking down the subendothelial basement membrane. Monocytes isolated from Cat $S^{-/-} L D L R^{-/-}$mice did not transmigrate the endothelial monolayer overlying a layer of type I and type IV collagen (Figure 5, $\mathrm{d}$ and $\mathrm{f}$ ), suggesting a role for Cat $\mathrm{S}$ in blood-borne leukocyte penetration through the arterial basement membrane after endothelial adhesion. Although we currently cannot define a clear mechanism for this process, Cat $\mathrm{S}$ may play multiple roles including ECM degradation, monocyte differentiation, and possibly even "reverse transmigration." Primary cultured monocytes from $\mathrm{CatS}^{-/-}$and control mice did not show significantly different invasion/transmigration in a collagen-coated Boyden chamber, suggesting that Cat S-mediated collagenolysis may not be the only factor affecting monocyte basement membrane transmigration. Monocyte immunostaining with F4/80, a marker for both monocytes and macrophages, indicated that most of the F4/80-positive cells from CatS $S^{-/} L D L R^{-/-}$mice are much smaller in size (and are possibly monocytes) (Figure $5 \mathrm{~d}$ ) than those (possibly macrophages) from control mice (Figure 5, $c$ and e). Therefore Cat $S$ may also affect monocyte differentiation in this context, although we lack direct evidence on this point. During atherogenesis, monocytes also traverse endothelium in the basal-to-apical direction (reverse transmigration) (47). Randolph et al. have shown that endogenous signals can stimulate monocyte traversal across an endothelial monolayer atop collagen matrix and that a significant fraction of these cells becomes dendritic cells after reverse transmigration (48). Cat S may thus affect monocyte reverse transmigration, although this issue requires further investigation.

Our observations did not suggest a role for Cat $\mathrm{S}$ in leukocyte recruitment as none of the adhesion or chemoattractant molecules measured in lesions showed differences between the two groups of mice (Table 2, 8-week timepoint). Impaired leukocyte transmigration at the early stage of lesion development and/or reduced levels of cell adhesion molecule (i.e., VCAM) and chemoattractant (i.e., MCP-1) in lesions at later stages in $\mathrm{CatS}^{-/-} L D L R^{-/-}$mice (Table 2) may account for the reduced number of lesional $\mathrm{CD}^{+} \mathrm{T}$ cells and macrophages and the reduced amount of IFN- $\gamma$ (Figure 4). In particular, MCP-1, which was most affected by Cat $\mathrm{S}$ deficiency, plays a critical role in monocyte-macrophage recruitment and migration (49). However, the monocyte recruitment/transmigration defect observed in $\mathrm{CatS}^{-/-}$ monocytes (Figure 5) may not be the major cause of the retarded atherosclerosis observed in this study. Indeed, MCP-1 levels declined strikingly at both the 12-week and 26-week timepoints (Table 2), in parallel with diminished lesion macrophage content (Figure 4 , a and b). Deeper understanding of the importance of MCP-1 participation in this setting may require further experiments such as bone marrow transplantation.

Cat S may also contribute to atherogenesis by effects on formation and growth of neovessels. Microvessels may influence plaque growth and lesion stability (50). Administration of the angiogenic factor VEGF was shown to significantly augment the experimental atheroma (51). In contrast, atherosclerotic animals treated with inhibitors of angiogenesis showed not only reduced intimal neovascularization but also reduced plaque size (52). Our recent observations suggested that Cat $S$ may contribute to the degradation of arterial ECM, which provides a path for neovessel growth (53). Cat $\mathrm{S}$ deficiency reduces microtubule formation in vitro and microvessel growth in vivo (G.-P. Shi et al., unpublished data). Therefore, the impaired microvessel growth observed in $\mathrm{CatS}^{-/-}$mice might limit atherosclerotic plaque growth.

In addition, we and others have previously reported that Cat $S$ plays an important role in invariant chain processing in antigen-presenting cells, which leads to altered antibody class switching and production $(20,21)$. Increased serum autoantibodies against oxidized LDL correlate with atherosclerosis in humans and animals $(54,55)$. Cat $\mathrm{S}$ deficiency may cause impaired immune responses and $\mathrm{T}$ lymphocyte activation and expansion. Indeed, the number of $\mathrm{T}$ lymphocytes also decreased markedly in lesions of Cat $S^{-/-} L D L R^{-/-}$mice. Further, CD1 molecules mediate presentation of lipid antigen to $\mathrm{T}$ cells and further $\mathrm{T}$ cell activation by lipid-laden macrophages in 
human atheroma. $\mathrm{CD} 1^{+}$foamy macrophages congregate with $\mathrm{T}$ cells in atherosclerotic plaques (56). $\mathrm{CatS}^{-/-}$mice have defective CD1-restricted antigen presentation (57), consistent with a role for this protease in antigen presentation by $\mathrm{CD} 1^{+}$macrophages. Therefore, altered immune responses found in $\mathrm{CatS}^{-/-}$ mice may also contribute to the retarded atherogenesis in $\mathrm{CatS}^{-/-} \mathrm{LDLR}^{-/-}$mice.

In conclusion, this study provides direct in vivo evidence for involvement of the cysteine protease Cat $\mathrm{S}$ in atherogenesis in mice. The data presented support several possible mechanisms that contribute to this atherogenic role of Cat $\mathrm{S}$. Our previous observations in diseased human arterial tissue suggest a similar function for this protease in human lesions. The present findings firmly place this cysteine protease alongside MMPs and serine proteases as proteolytic enzymes involved in atherosclerosis. This work raises numerous questions for future investigation and underscores the need to consider Cat $\mathrm{S}$ as a therapeutic target in arterial diseases.

\section{Acknowledgments}

The authors thank Harold A. Chapman and Karen Williams for reading the manuscript, and Eugenia Shvartz, Kenji Ohyauchi, Susumu Momozaki, Mika Kobayashi, and Akashi Izumi for their excellent technical assistance. This work is supported by grants from the NIH National Heart, Lung, and Blood Institute to G.-P. Shi (HL-60942) and P. Libby (HL-34636 and SCOR grant HL-56985).

1. Sukhova, G.K., Shi, G.P., Simon, D.I., Chapman, H.A., and Libby, P. 1998 Expression of the elastolytic cathepsins $\mathrm{S}$ and $\mathrm{K}$ in human atheroma and regulation of their production in smooth muscle cells. J. Clin. Invest. 102:576-583.

2. Shi, G.P., et al. 1999. Cystatin C deficiency in human atherosclerosis and aortic aneurysms. J. Clin. Invest. 104:1191-1197.

3. Libby, P. 2000. Changing concepts of atherogenesis. J. Intern. Med. 247:349-358.

4. Moons, L., et al. 1998. Reduced transplant arteriosclerosis in plasminogen-deficient mice. J. Clin. Invest. 102:1788-1797.

5. Carmeliet, P., Moons, L., Ploplis, V., Plow, E., and Collen, D. 1997. Impaired arterial neointima formation in mice with disruption of the plasminogen gene. J. Clin. Invest. 99:200-208

6. Carmeliet, P., et al. 1997. Urokinase-generated plasmin activates matrix metalloproteinases during aneurysm formation. Nat. Genet. 17:439-444.

7. Galis, Z.S., Sukhova, G.K., Lark, M.W., and Libby, P. 1994. Increased expression of matrix metalloproteinases and matrix degrading activity in vulnerable regions of human atherosclerotic plaques. J. Clin. Invest. 94:2493-2503.

8. Sukhova, G.K., et al. 1999. Evidence for increased collagenolysis by interstitial collagenases- 1 and -3 in vulnerable human atheromatous plaques. Circulation. 99:2503-2509.

9. Herman, M.P., et al. 2001. Tissue factor pathway inhibitor-2 is a novel inhibitor of matrix metalloproteinases with implications for atherosclerosis. J. Clin. Invest. 107:1117-1126.

10. Brown, D.L., Hibbs, M.S., Kearney, M., Loushin, C., and Isner, J.M. 1995 Identification of $92-\mathrm{kD}$ gelatinase in human coronary atherosclerotic lesions. Association of active enzyme synthesis with unstable angina. Circulation. 91:2125-2131.

11. Henney, A.M., et al. 1991. Localization of stromelysin gene expression in atherosclerotic plaques by in situ hybridization. Proc. Natl. Acad. Sci. U. S. A. 88:8154-8158.

12. Halpert, I., et al. 1996. Matrilysin is expressed by lipid-laden macrophages at sites of potential rupture in atherosclerotic lesions and localizes to areas of versican deposition, a proteoglycan substrate for the enzyme. Proc. Natl. Acad. Sci. U. S. A. 93:9748-9753.

13. Jormsjo, S., et al. 2000. Allele-specific regulation of matrix metallopro- teinase-12 gene activity is associated with coronary artery luminal dimensions in diabetic patients with manifest coronary artery disease. Circ. Res. 86:998-1003.

14. Hiller, O., Lichte, A., Oberpichler, A., Kocourek, A., and Tschesche, H. 2000. Matrix metalloproteinases collagenase-2, macrophage elastase, collagenase-3, and membrane type 1-matrix metalloproteinase impair clotting by degradation of fibrinogen and factor XII. J. Biol. Chem. 275:33008-33013.

15. Allaire, E., Forough, R., Clowes, M., Starcher, B., and Clowes, A.W. 1998. Local overexpression of TIMP-1 prevents aortic aneurysm degeneration and rupture in a rat model. J. Clin. Invest. 102:1413-1420.

16. Rouis, M., et al. 1999. Adenovirus-mediated overexpression of tissue inhibitor of metalloproteinase- 1 reduces atherosclerotic lesions in apolipoprotein E-deficient mice. Circulation. 100:533-540.

17. Xin, X.Q., Gunesekera, B., and Mason, R.W. 1992. The specificity and elastinolytic activities of bovine cathepsins $\mathrm{S}$ and $\mathrm{H}$. Arch. Biochem. Biophys. 299:334-339.

18. Shi, G.P., Munger, J.S., Meara, J.P., Rich, D.H., and Chapman, H.A. 1992. Molecular cloning and expression of human alveolar macrophage cathepsin S, an elastinolytic cysteine protease. J. Biol. Chem. 267:7258-7262.

19. Maciewicz, R.A., and Etherington, D.J. 1998. A comparison of four cathepsins (B, L, N and S) with collagenolytic activity from rabbit spleen. Biochem. J. 256:433-440.

20. Shi, G.P., et al. 1999. Cathepsin S required for normal MHC class II peptide loading and germinal center development. Immunity. 10:197-206.

21. Nakagawa, T.Y., et al. 1999. Impaired invariant chain degradation and antigen presentation and diminished collagen-induced arthritis in cathepsin S null mice. Immunity. 10:207-217.

22. Hansson, G.K. 2001. Regulation of immune mechanisms in atherosclerosis. Ann. N. Y. Acad. Sci. 947:157-165.

23. Robbie, L., and Libby, P. 2001. Inflammation and atherothrombosis. Ann. N. Y. Acad. Sci. 947:167-179.

24. Ishibashi, S., et al. 1993. Hypercholesterolemia in low density lipoprotein receptor knockout mice and its reversal by adenovirus-mediated gene delivery. J. Clin. Invest. 92:883-893.

25. Lichtman, A.H., et al. 1999. Hyperlipidemia and atherosclerotic lesion development in LDL receptor-deficient mice fed defined semipurified diets with and without cholate. Arterioscler. Thromb. Vasc. Biol. 19:1938-1944.

26. Ray, J.L., Leach, R., Herbert, J.M., and Benson, M. 2001. Isolation of vascular smooth muscle cells from a single murine aorta. Methods Cell Sci. 23:185-188.

27. Mach, F., Schonbeck, U., Sukhova, G.K., Atkinson, E., and Libby, P. 1998. Reduction of atherosclerosis in mice by inhibition of CD40 signaling. Nature. 394:200-203.

28. Takaku, M., et al. 1999. An in vitro coculture model of transmigrant monocytes and foam cell formation. Arterioscler. Thromb. Vasc. Biol. 19:2330-2339.

29. Austyn, J.M., and Gordon, S. 1981. F4/80, a monoclonal antibody directed specifically against the mouse macrophage. Eur. J. Immunol. 11:805-815.

30. Honda, M., et al. 1998. Immunohistochemical evidence for a macrophage scavenger receptor in Mato cells and reactive microglia of ischemia and Alzheimer's disease. Biochem. Biophys. Res. Commun. 245:734-740

31. Ishibashi, S., Goldstein, J.L., Brown, M.S., Herz, J., and Burns, D.K. 1994. Massive xanthomatosis and atherosclerosis in cholesterol-fed low density lipoprotein receptor-negative mice. J. Clin. Invest. 93:1885-1893.

32. Davies, M.J., Richardson, P.D., Woolf, N., Katz, D.R., and Mann, J. 1993. Risk of thrombosis in human atherosclerotic plaques: role of extracellular lipid, macrophage, and smooth muscle cell content. Br. Heart J. 69:377-381.

33. Roselaar, S.E., Kakkanathu, P.X., and Daugherty, A. 1996. Lymphocyte populations in atherosclerotic lesions of $\mathrm{apoE}^{-/-}$and LDL receptor $-/$ mice. Decreasing density with disease progression. Arterioscler. Thromb. Vasc. Biol. 16:1013-1018.

34. Hansson, G.K., Libby, P., Schonbeck, U., and Yan, Z.Q. 2002. Innate and adaptive immunity in the pathogenesis of atherosclerosis. Circ. Res. 91:281-291.

35. Huo, Y., et al. 2001. The chemokine KC, but not monocyte chemoattractant protein-1, triggers monocyte arrest on early atherosclerotic endothelium. J. Clin. Invest. 108:1307-1314. doi:10.1172/JCI200112877.

36. Libby, P., Sukhova, G., Lee, R.T., and Galis, Z.S. 1995. Cytokines regulate vascular functions related to stability of the atherosclerotic plaque. J. Cardiovasc. Pharmacol. 25(Suppl. 2):S9-S12.

37. Farb, A., et al. 1996. Coronary plaque erosion without rupture into a lipid core. A frequent cause of coronary thrombosis in sudden coronary death. Circulation. 93:1354-1363.

38. Libby, P., Ganz, P., Schoen, F.J., and Lee, R.T. 2000. The vascular biology of the acute coronary syndromes. In Acute coronary syndromes. E.J. Topol, editor. Marcel Dekker Inc. New York, New York, USA. 33-57. 
39. Maciewicz, R.A., and Etherington, D.J. 1988. A comparison of four cathepsins (B, L, N and S) with collagenolytic activity from rabbit spleen. Biochem. J. 256:433-440.

40. Chapman, H.A., Riese, R.J., and Shi, G.-P. 1997. Emerging roles for cysteine proteases in human biology. Annu. Rev. Physiol. 59:63-88.

41. Reddy, V.Y., Zhang, Q.Y., and Weiss, S.J. 1995. Pericellular mobilization of the tissue-destructive cysteine proteinases, cathepsins B, L, and S, by human monocyte-derived macrophages. Proc. Natl. Acad. Sci. U. S. A. 92:3849-3853.

42. Punturieri, A., et al. 2000. Regulation of elastinolytic cysteine proteinase activity in normal and cathepsin K-deficient human macrophages. J. Exp. Med. 192:789-799.

43. De Meyer, G.R., and Bult, H. 1997. Mechanisms of neointima formation-lessons from experimental models. Vasc. Med. 2:179-189.

44. Li, D.Y., et al. 1998. Elastin is an essential determinant of arterial morphogenesis. Nature. 393:276-280.

45. Amento, E.P., Ehsani, N., Palmer, H., and Libby, P. 1991. Cytokines and growth factors positively and negatively regulate interstitial collagen gene expression in human vascular smooth muscle cells. Arterioscler. Thromb. 11:1223-1230.

46. Murray, K., de Lera, J.M., Astudillo, A., and McNicol, A.M. 1997. Organisation of basement membrane components in the human adult and fetal pituitary gland and in pituitary adenomas. Virchows Arch. 431:329-335.

47. Randolph, G.J., Luther, T., Albrecht, S., Magdolen, V., and Muller, W.A. 1998. Role of tissue factor in adhesion of mononuclear phagocytes to and trafficking through endothelium in vitro. Blood. 92:4167-4177.

48. Randolph, G.J., Sanchez-Schmitz, G., Liebman, R.M., and Schakel, K.
2002. The CD16(+) (FcgammaRIII(+)) subset of human monocytes preferentially becomes migratory dendritic cells in a model tissue setting. J. Exp. Med. 196:517-527.

49. Valente, A.J., Rozek, M.M., Sprague, E.A., and Schwartz, C.J. 1992. Mechanisms in intimal monocyte-macrophage recruitment. A special role for monocyte chemotactic protein-1. Circulation. 86(Suppl. 6):III20-III25.

50. Moulton, K.S. 2001. Plaque angiogenesis and atherosclerosis. Curr. Atheroscler. Rep. 3:225-233.

51. Celletti, F.L., et al. 2001. Vascular endothelial growth factor enhances atherosclerotic plaque progression. Nat. Med. 7:425-429.

52. Moulton, K.S., et al. 1999. Angiogenesis inhibitors endostatin or TNP470 reduce intimal neovascularization and plaque growth in apolipoprotein E-deficient mice. Circulation. 99:1726-1732.

53. Libby, P., and Schonbeck, U. 2001. Drilling for oxygen: angiogenesis involves proteolysis of the extracellular matrix. Circ. Res. 89:195-197.

54. Bui, M.N., et al. 1996. Autoantibody titers to oxidized low-density lipoprotein in patients with coronary atherosclerosis. Am. Heart J. 131:663-667.

55. Palinski, W., Tangirala, R.K., Miller, E., Young, S.G., and Witztum, J.L. 1995. Increased autoantibody titers against epitopes of oxidized LDL in LDL receptor-deficient mice with increased atherosclerosis. Arterioscler. Thromb. Vasc. Biol. 15:1569-1576.

56. Melian, A., Geng, Y.J., Sukhova, G.K., Libby, P., and Porcelli, S.A. 1999. $\mathrm{CD} 1$ expression in human atherosclerosis. A potential mechanism for $\mathrm{T}$ cell activation by foam cells. Am. J. Pathol. 155:775-786

57. Riese, R.J., et al. 2001. Regulation of CD1 function and NK1.1(+) T cell selection and maturation by cathepsin S. Immunity. 15:909-519. 